\title{
CHR: DYNAMIC FunCTIONAL CONSTRAINTS CheCKING IN R
}

\author{
Konrad Grzanek \\ IT Institute, University of Social Sciences \\ 9 Sienkiewicza St., 90-113 Lodz, Poland \\ kgrzanek@spoleczna.pl
}

\begin{abstract}
Dynamic typing of R programming language may issue some quality problems in large scale data-science and machine-learning projects for which the language is used. Following our efforts on providing gradual typing library for Clojure we come with a package chR - a library that offers functionality of run-time typerelated checks in R. The solution is not only a dynamic type checker, it also helps to systematize thinking about types in the language, at the same time offering high expressivenes and full adherence to functional programming style.
\end{abstract}

Keywords: Formal software verification, software quality, dynamic type-checking, functional programming, category theory, $\mathrm{R}$

\section{Introduction}

Writing software in dynamically typed programming languages requires as much attention with respect to types of expressions as when using a statically and strongly typed one ([2], [1]). One popular and apparently natural approach is to use gradual typing - a process of selectively adding type checks to expressions, mostly to the critical parts of computer programs. With the approach a programmer can decide where to put checks and which parts are so "obvious", that they do not have to be verified.

$\mathrm{R}$ programming language [5] has been growing in use in recent years, together with a growth of computer science and software engineering sub-domains to which it has been targeted: data science and statistical- (more broadly machine-) learning. Unfortunately, it lacks a decent type-checking solution. The great assertthat package [7] addresses a slightly different problem: putting generic run-time assertions into $\mathrm{R}$ codes. We need a package with the following properties:

- being deeply rooted in functional programming [4] and using notions from the category theory 
- overall consistency with the dynamic and in a way Lispy nature of R programming language and adherence to functional programming style with purrr library [6]

- being as fast as possible, using checks that use as low-level elements of the R base (standard library) as possible

- expressiveness, ease of use, and extendability

Our previous work on dependent typing resulted in a ch library ([11], [12], [13]) for Clojure programming language ([8], [9]). Following that we decided to create a corresponding package for $\mathrm{R}$. The package is called $\operatorname{ch} R[10]$ and it is a subject of further analysis in this paper.

\section{Essentials of the chR Library}

The heart of our solution is $c h$ procedure. In essence it executes a predicate (pred) on an argument $x$. If the predicate returns false value (or a value effectively) effectively equal to false, an error is raised. Otherwise $x$ is returned. This behavior allows a greater composability and support for functional programming style. We may easily put any ch(eck) in a pipeline of data processing procedures, as will be presented further.

Procedure $c h$ works also in a predicate-only mode. This mode is necessary when a $\mathrm{ch}(\mathrm{eck})$ is used as a sub-component of a larger one. Below we have the ch code together with an error generator (errMessage):

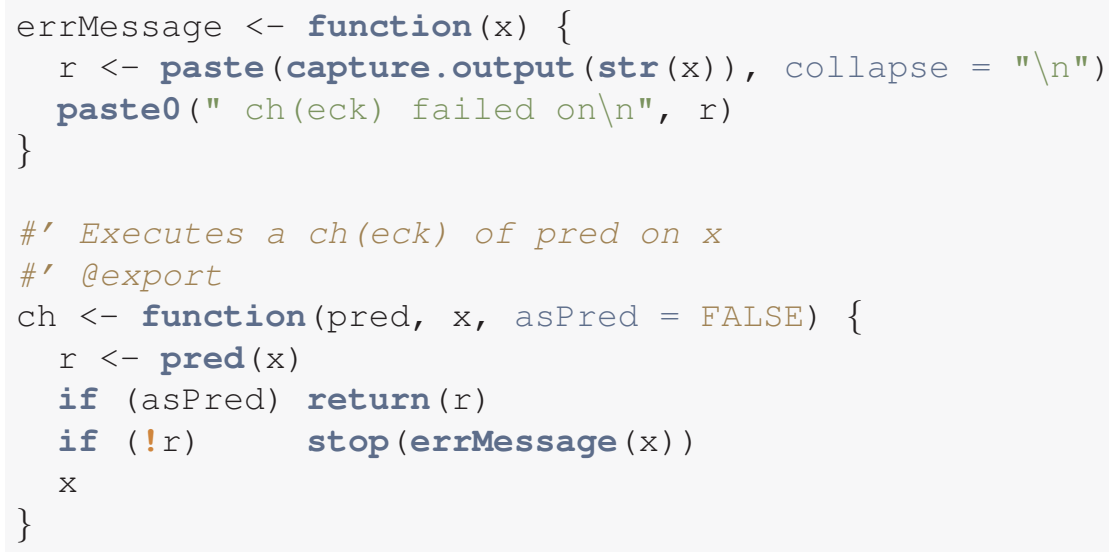

Effective use of $c h R$ library starts with the following procedure that takes a predicate and returns a corresponding ch(eck). The returned ch(eck) takes an argument $x$ and applies $c h$ working by default in non-pred mode:

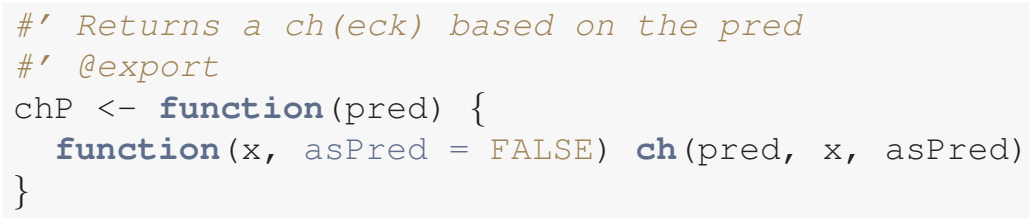


For classes in $\mathrm{R}$ we have a chInstance ch(eck)s generator that uses a common inherits procedure belonging to $\mathrm{R}$ base:

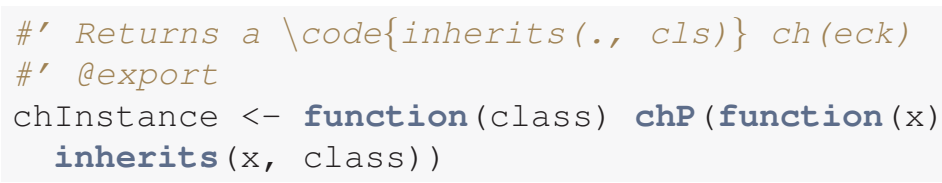

An intrinsic property of an expressive language (including an embedded one) is composability. Our ch(eck)s compose. The three composition operators are logicoriented and they reflect the most basic logical operations. We have negation:

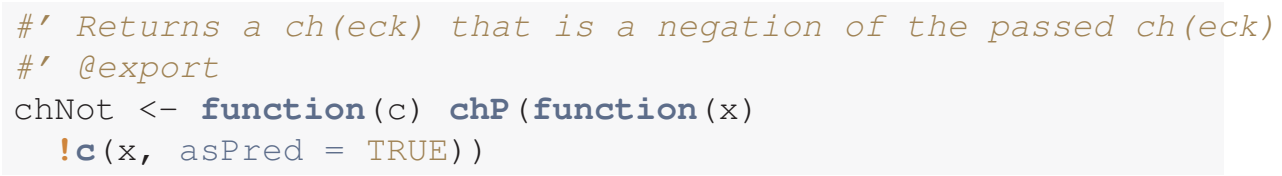

Also, there is conjunction:

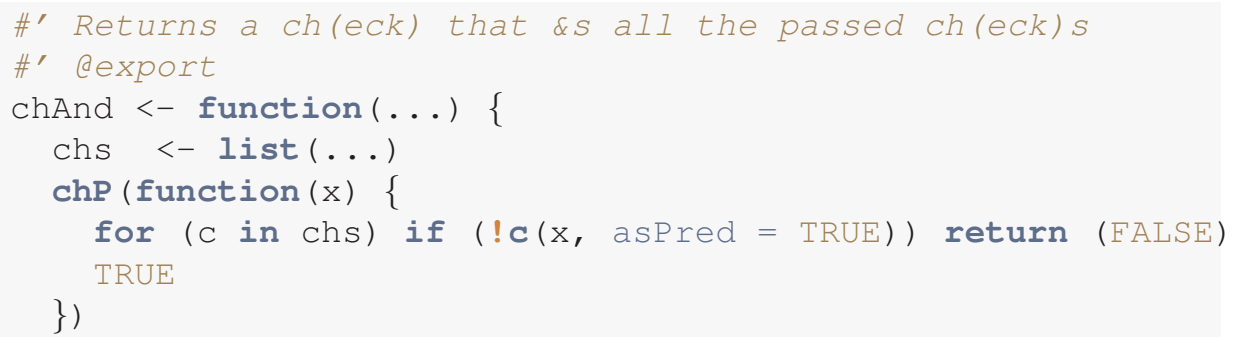

and alternative:

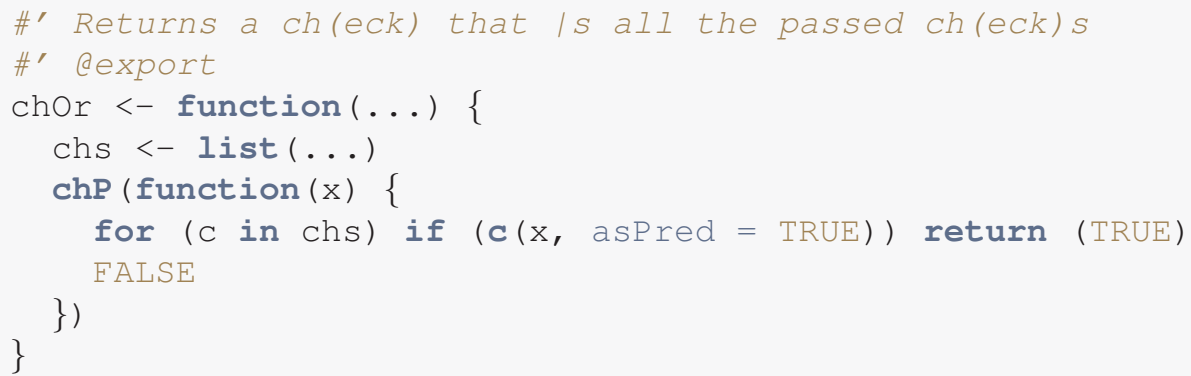

In the two latter ones we assume a non-restricted number of composed ch(eck)s.

\section{Fundamental $\mathrm{Ch}(\mathrm{eck}) \mathrm{s}$}

Many functional programming languages rooted in category theory ([3]), e.g. Haskell, use a unit type and unit value. Although R fully supports functional style of program- 
ming, it does not unify notion of no-values. We made an arbitrary decision to treat NULL as unit value. The decision was based upon pragmatics in the technology. A corresponding ch(eck) follows:

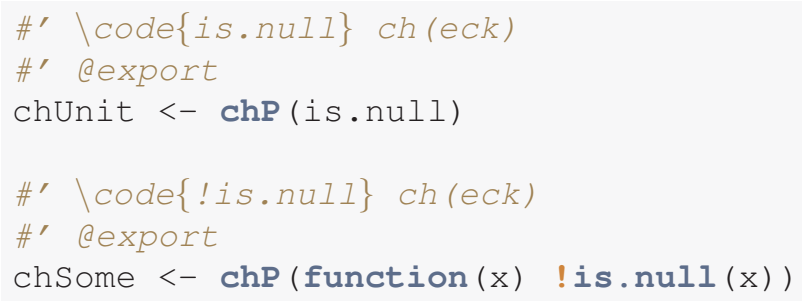

The chSome ch(eck) is an opposite to chUnit, as can be seen above. In R programming language we have both NULL as well as NA values. Thus, a separate ch(eck) for NAs is needed:

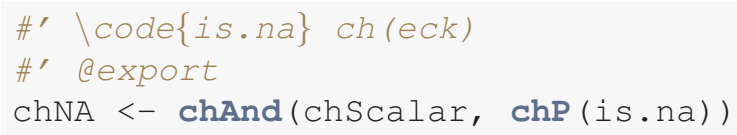

For two-element Discriminated Union Types we have the following chEither ch(eck):

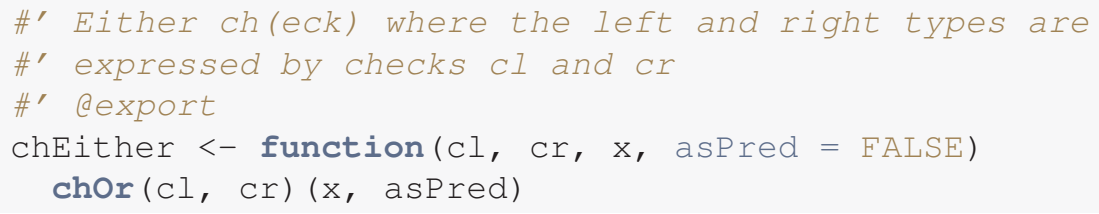

that used with chUnit forms a Maybe ch(eck):

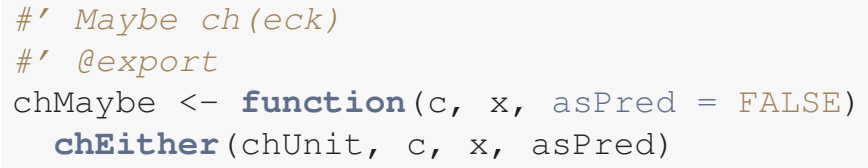

Because R uses only vectorized values (and lists), we need ch(eck)s for scalars, hereby treated as one-element atoms (vectors):

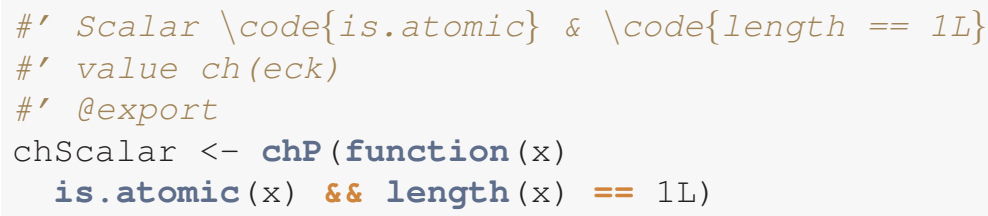

With the new ch(eck) we can define e.g. a ch(eck) for either a String vector of any length or a single String: 


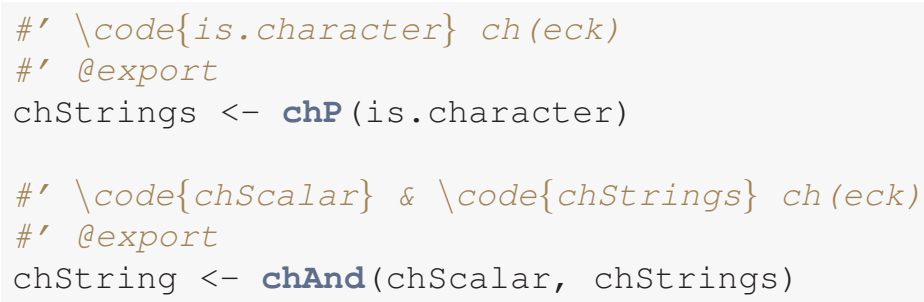

Another essential ch(eck) is for R functions:

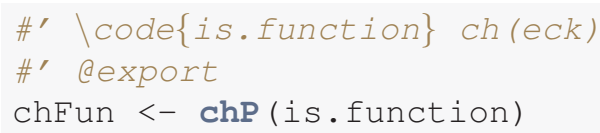

This shortened presentation ends a section about the most common ch(ecks)s in $c h R$ library. For more, please read Appendix A.

\section{Registry of Ch(eck)s}

Additionally the $c h R$ library (like its ancestor $c h$ for Clojure) provides a registry of $\mathrm{ch}(\mathrm{eck}) \mathrm{s}$, that helps the programmer to understand, what kind of ch(eck)s an object or a collection of objects fulfill. The registry is an associative container that can be used to put a relation between a ch(eck) symbol (name) and the ch(eck):

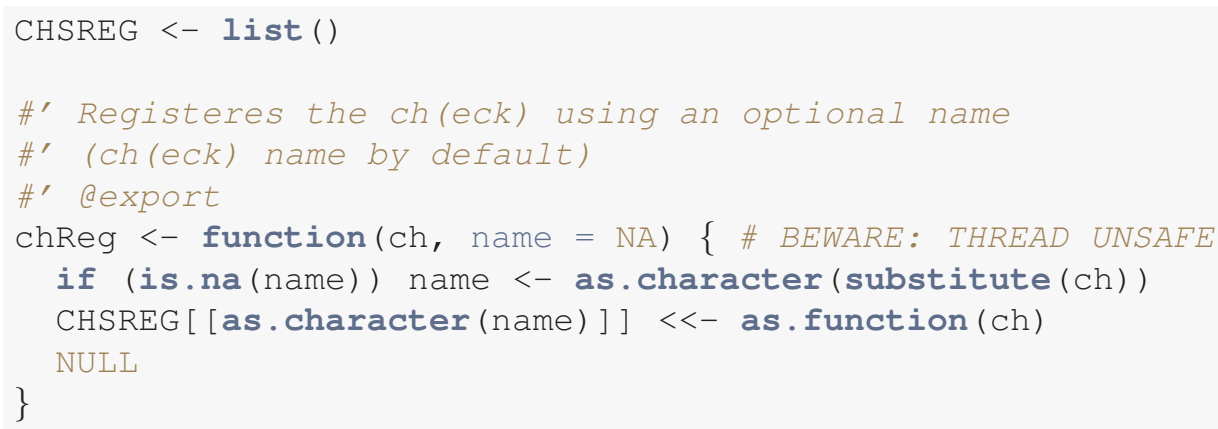

After we register selected ch(eck)s like below:

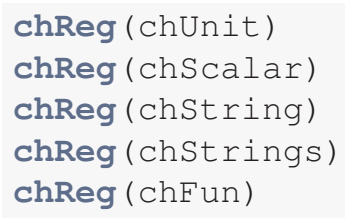

we can ask the library about what kinds of ch(eck)s a given object fulfills: 
$\operatorname{chR}:: \operatorname{chs}(1: 10)$

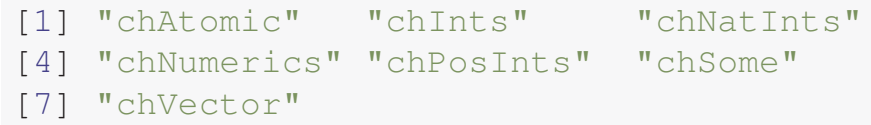

\section{Supporting $\mathrm{C}++$ Codes (via Rcpp)}

Some of the ch(eck)s are better implemented in a low-level programming language. Thankfully R supports easy extensions in C++ written in effective library Rcpp. In $c h R$ the following procedure is defined to allow evaluation of predicates on vectors of any (presumably numeric) types:

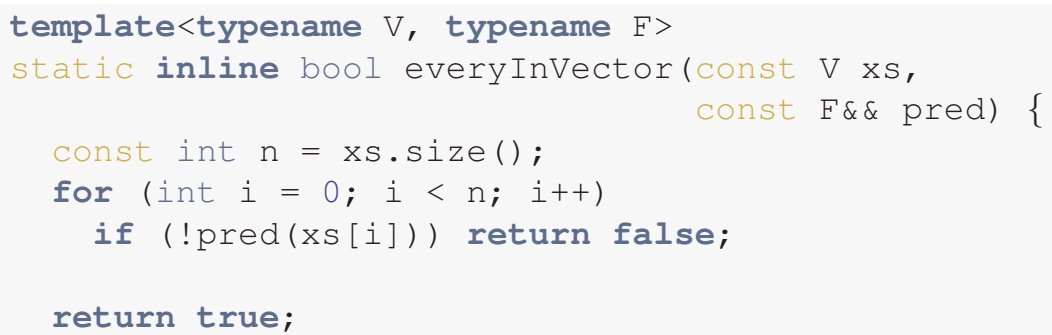

The procedure is used to implement the predicates on vectors of doubles, as presented below:

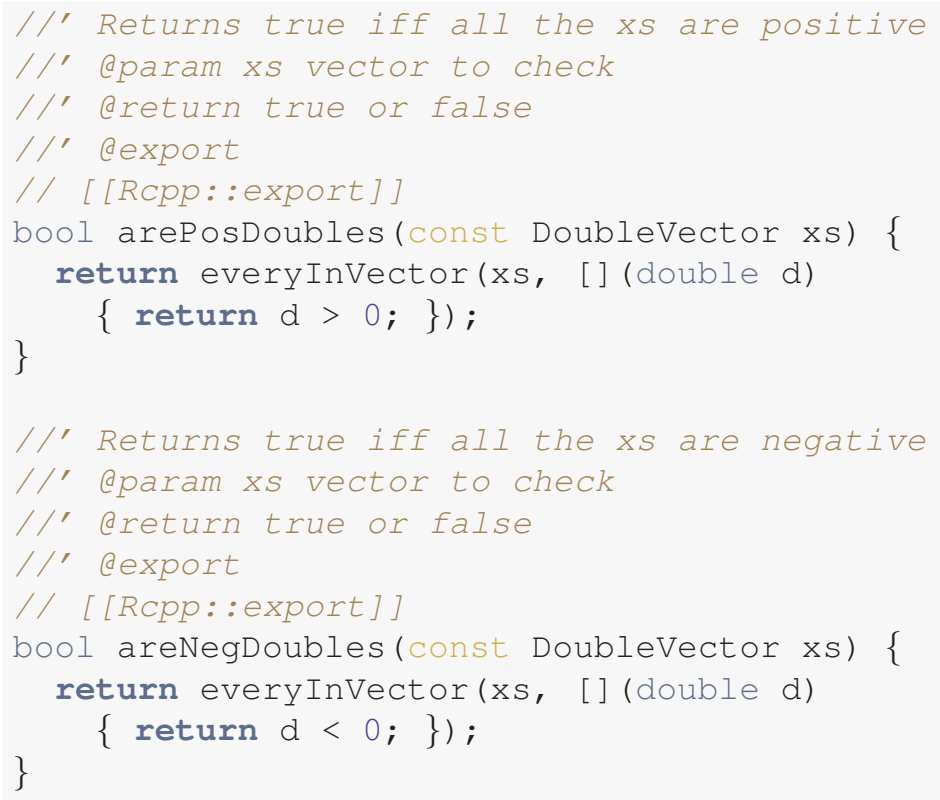




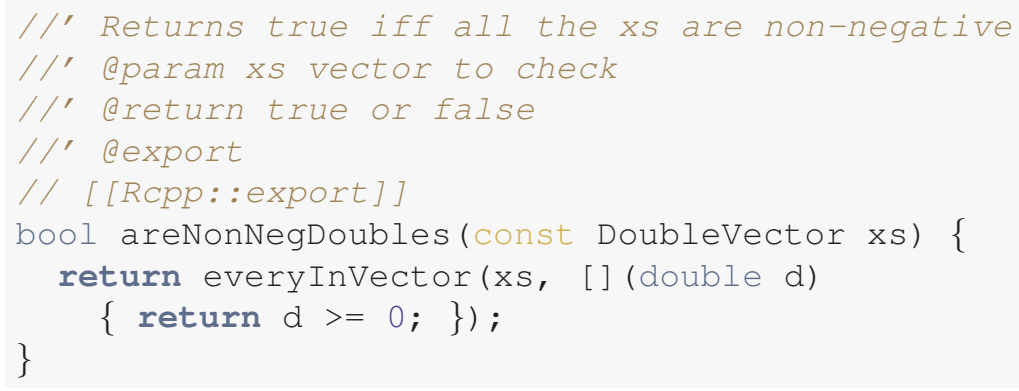

Accordingly, there are the preds for IntegerVector:

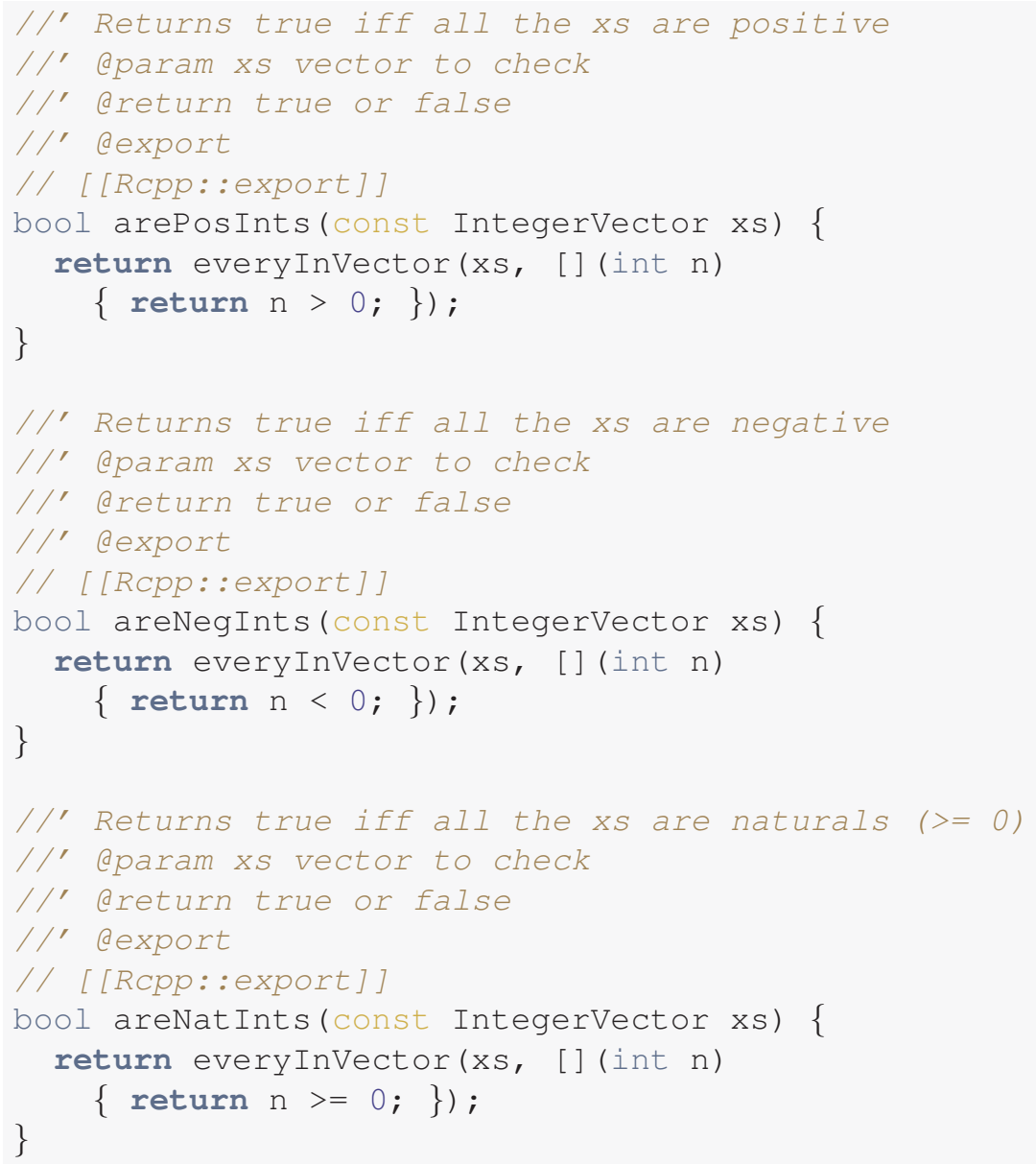

What's interesting is use of $\mathrm{C}++$ lambdas in the procedures above. They are nocost an highly expressive. Their use is possible in $\mathrm{C}++11$ and above. Current Rcpp implementation supports that language standard. 


\section{Cases of Use in Production Setting}

Our library is currently used in at least three commercial products. The usefulness of ch(eck)s can be seen in the following procedure, whose goal is to read employees' absences information in a business intelligence project:

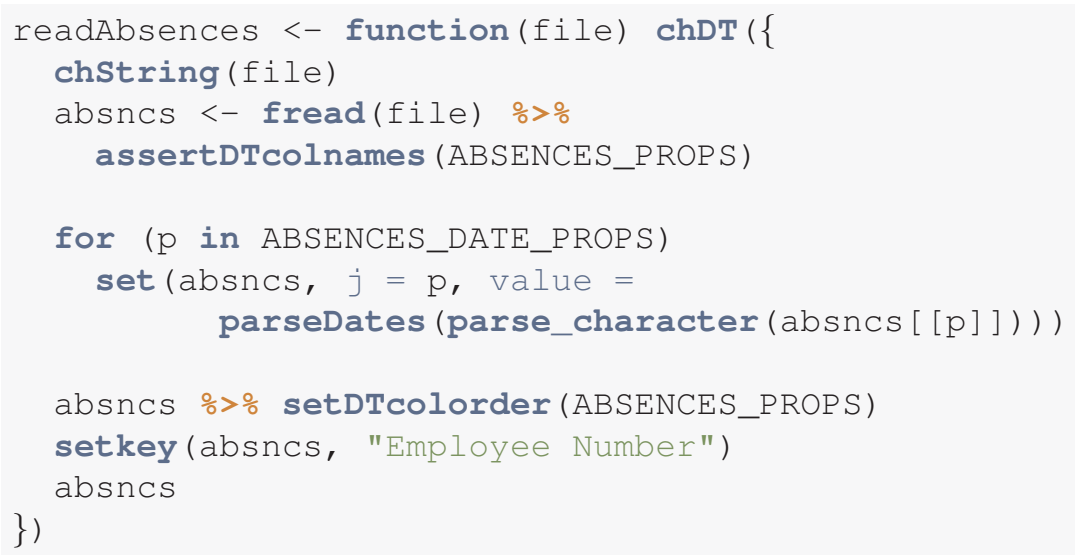

The argument file is intended to be a String (chString(file) ch(eck)) and the result of the procedure is a data.table object $(\operatorname{chDT}(\{\ldots\}) \operatorname{ch}(\mathrm{eck}))$. Apparently, the system of ch(eck)s not only increases software correctness, but also has a positive impact on readability of codes.

\section{References}

1. Pierce B.C., 2002, Types and Programming Languages, 1st Edition, MIT Press, ISBN-10: 0262162091, ISBN-13: 978-0262162098

2. Pierce B.C., 2004, Advanced Topics in Types and Programming Languages, MIT Press, ISBN-10: 0262162288, ISBN-13: 978-0262162289

3. Awodey S., 2010, Category Theory, Second Edition, Oxford University Press

4. Bird R., Wadler R., 1988, Introduction to Functional Programming. Series in Computer Science (Editor: C.A.R. Hoare), Prentice Hall International (UK) Ltd

5. R Core Team, 2017, R: A language and environment for statistical computing, $\mathrm{R}$ Foundation for Statistical Computing, Vienna, Austria, https://www.R-project.org/

6. Henry L., Wickham H., 2017, purrr: Functional Programming Tools, R package version 0.2.4, https://CRAN.R-project.org/package=purrr

7. Wickham H., 2017, assertthat: Easy Pre and Post Assertions, R package version 0.2.0, https://CRAN.R-project.org/package=assertthat 
8. Emerick Ch., Carper B., Grand Ch., 2012, Clojure Programming, O’Reilly Media Inc., ISBN: 978-1-449-39470-7

9. Fogus M., Houser Ch., 2014, The Joy of Clojure, Manning Publications; 2 edition, ISBN-10: 1617291412, ISBN-13: 978-1617291418

10. Grzanek K., 2017, chR Github Repository, https://github.com/kongra/chR

11. Grzanek K., 2016, Low-Cost Dynamic Constraint Checking for the JVM, Journal of Applied Computer Science Methods, 8(2), pp. 115-136, doi:10.1515/jacsm-20160008

12. Grzanek K., 2017, ch GitHub Repository, https://github.com/kongra/ch

13. Grzanek K., 2017, ch Clojars Page, https://clojars.org/kongra/ch

\section{A Appendix: Selected Pre-defined $\mathrm{Ch}($ eck)s}

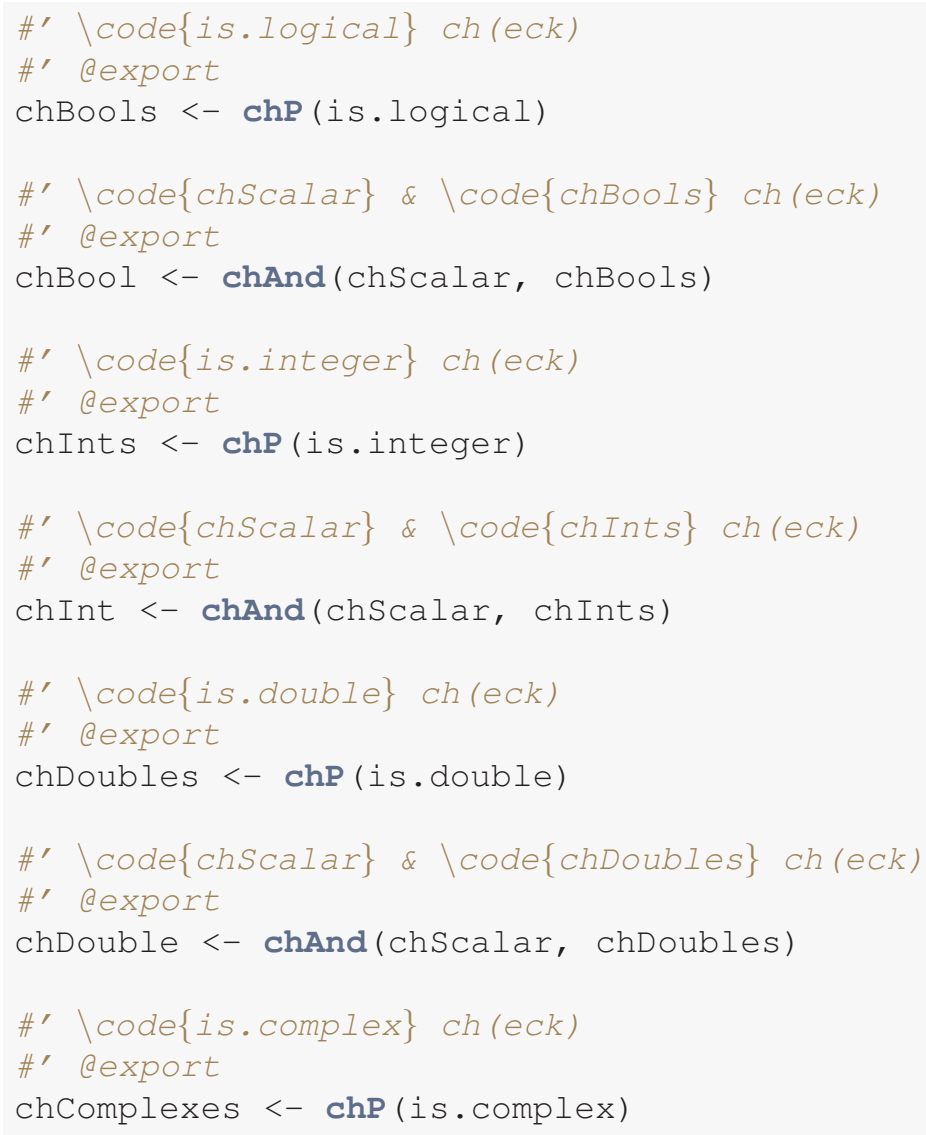




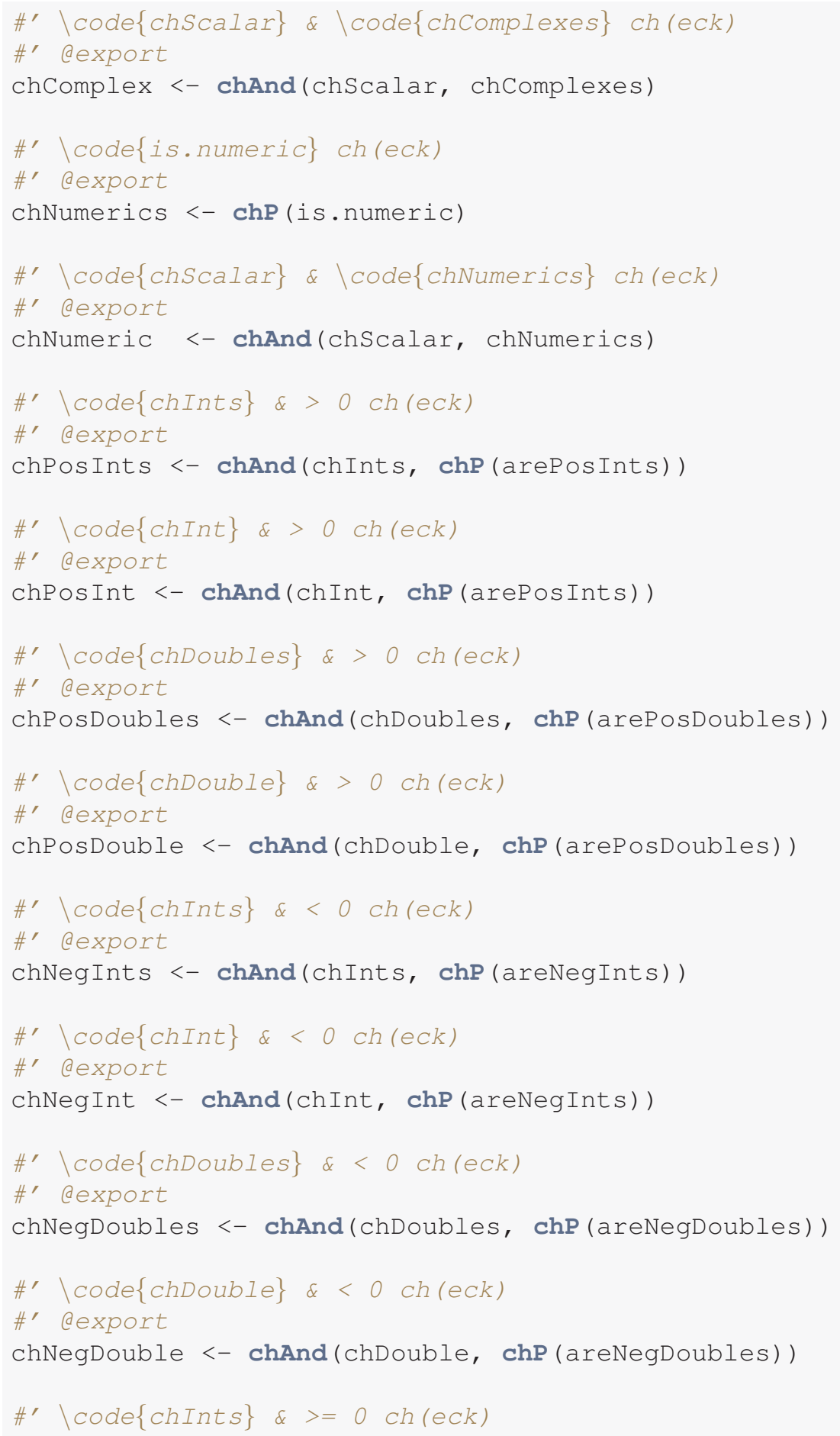




\section{Grzanek K.}

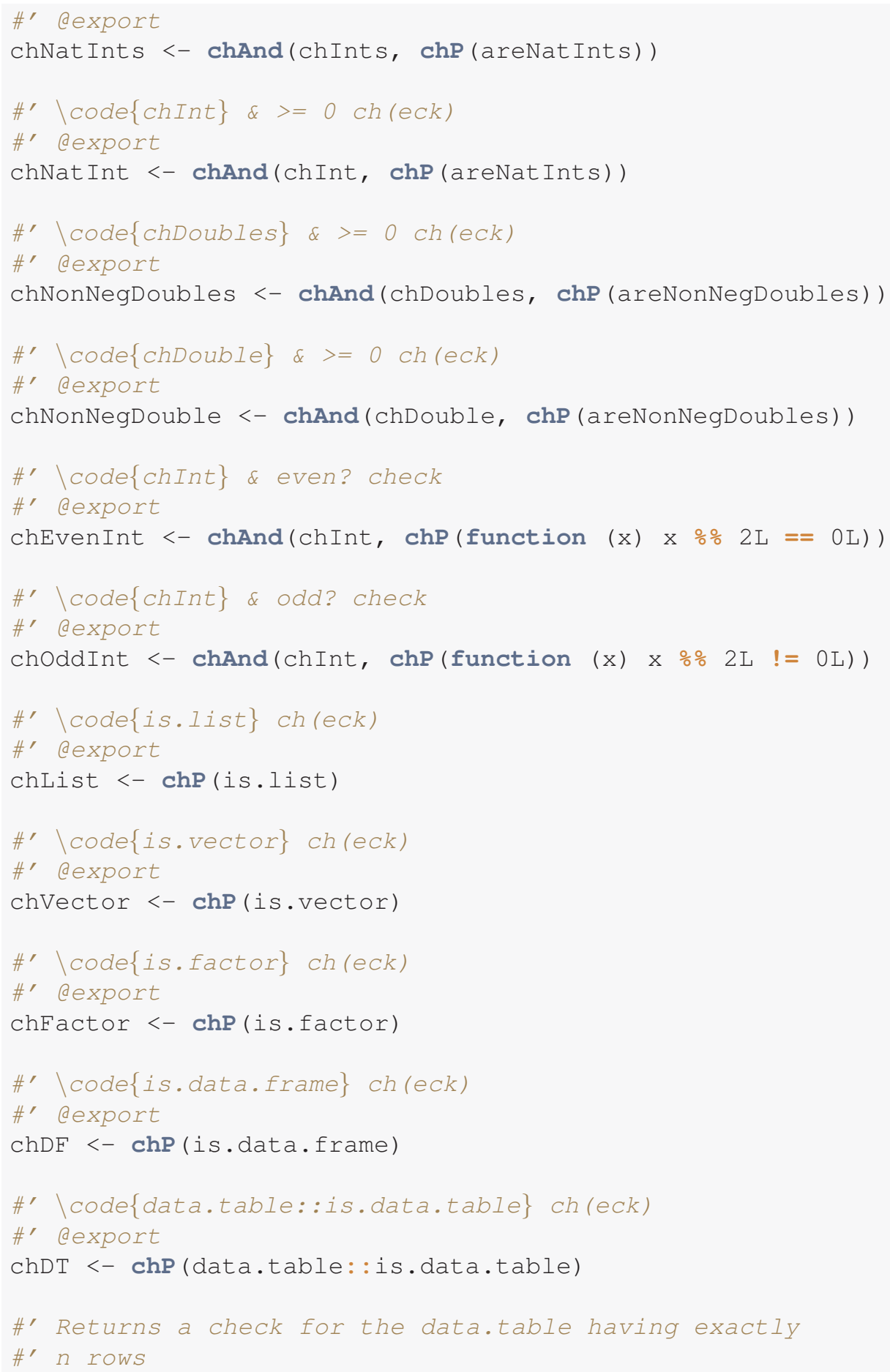




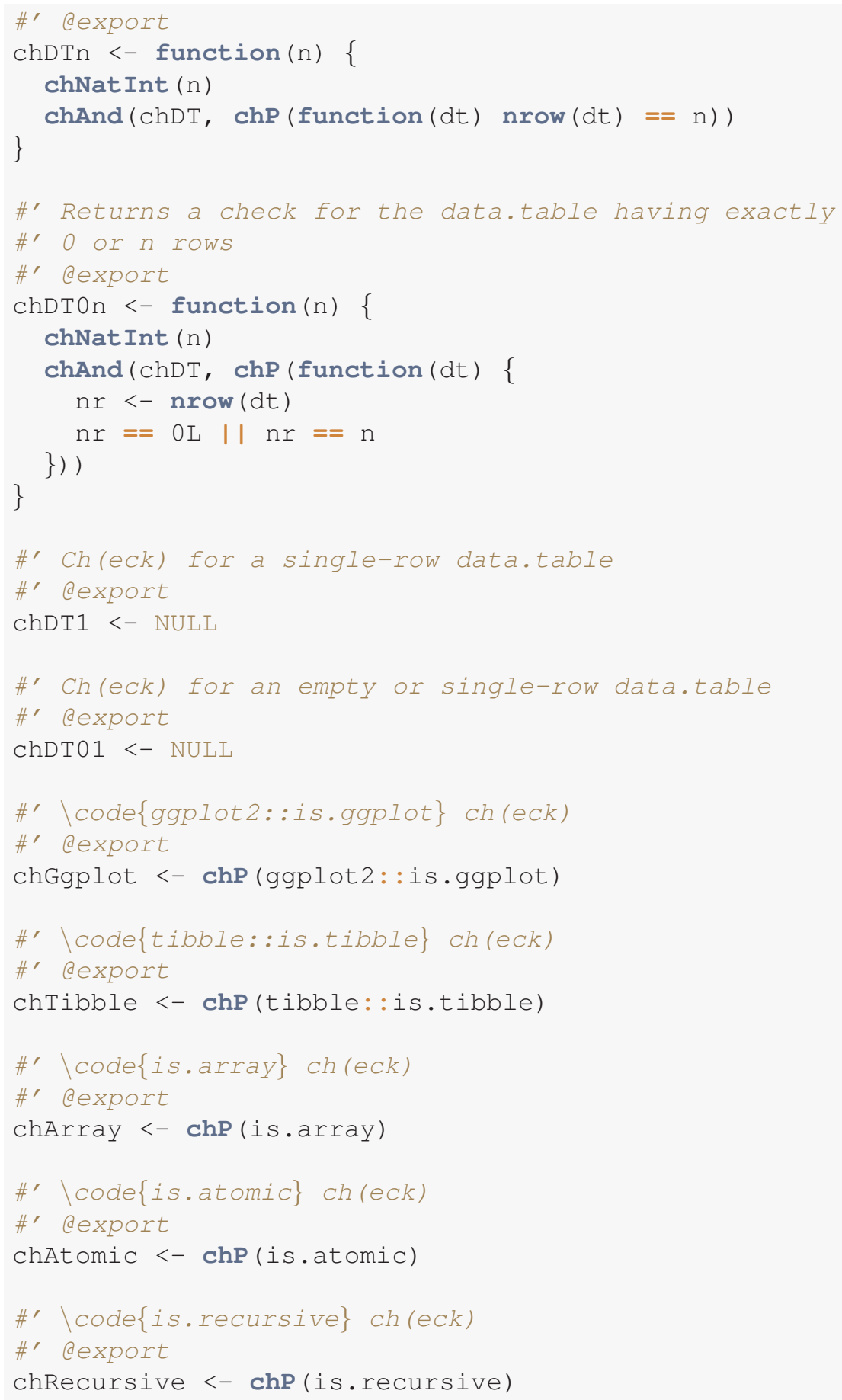




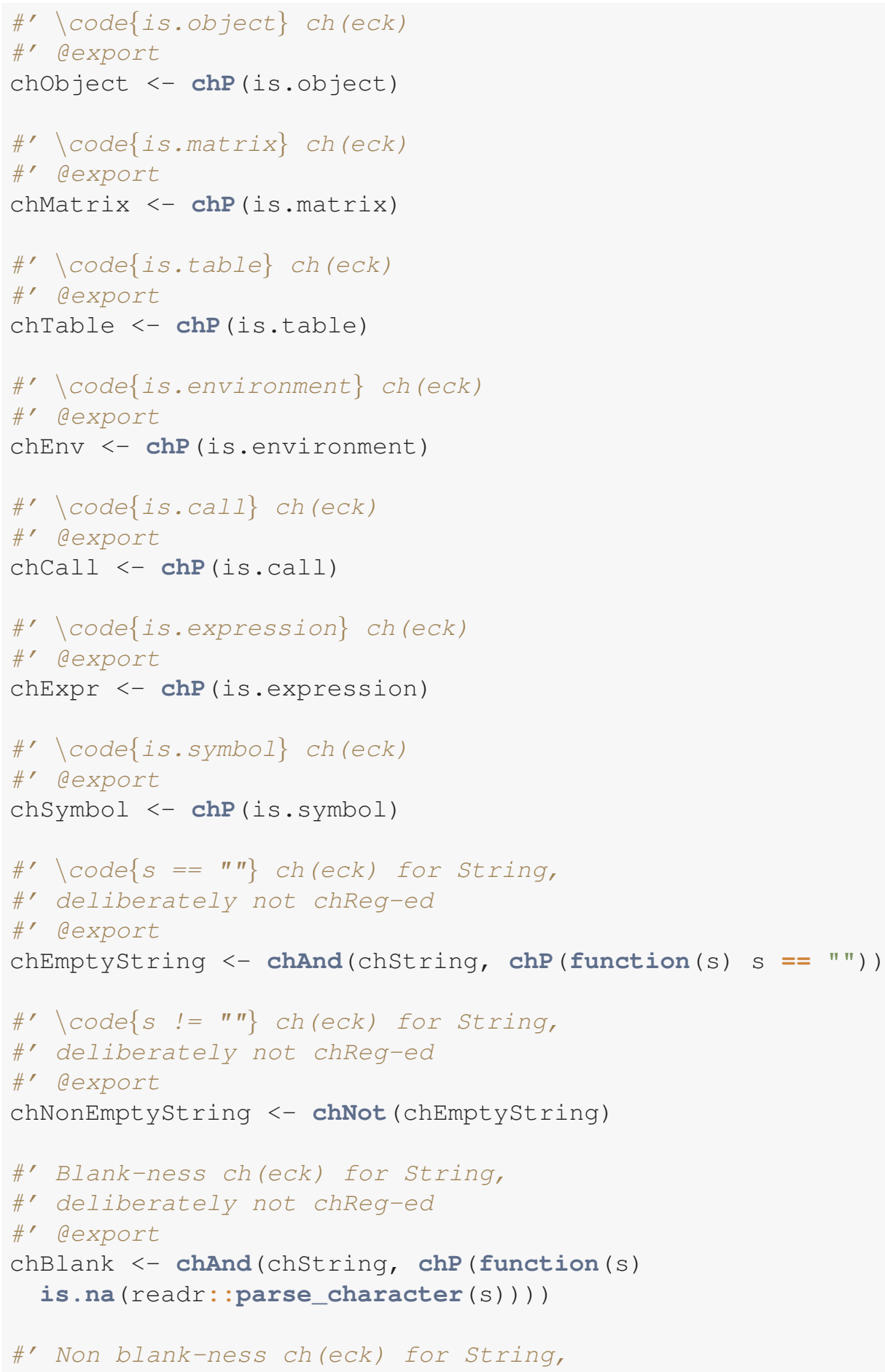




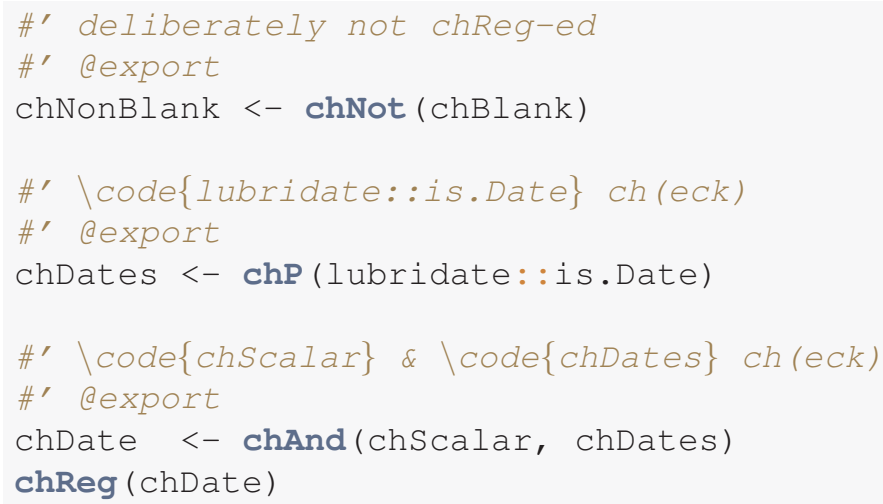

